\title{
A case of acute febrile neck pain
}

\author{
Daniele Cammelli ${ }^{1} \cdot$ Gianfranco Vitiello $^{2}$ (I) $\cdot$ Boaz Palterer $^{2} \cdot$ Andrea Rosi $^{3}$ • \\ Luciano Gabbani ${ }^{4}$
}

Received: 9 September 2016/ Accepted: 15 September 2016/Published online: 22 September 2016

(C) SIMI 2016

An 80-year-old woman presented with a 2-month history of worsening pain of her neck, associated with a 3-week history of hypomobility, and a progressive hyposthenia of the upper and the lower limbs. For this reason, she was admitted to our department. The past history was positive for osteoarthritis and hypertension. The patient was febrile (up to $39{ }^{\circ} \mathrm{C}$ ), and she complained about sharp severe neck pain causing rigidity and preventing neck movement. The physical examination revealed plegia of both upper limbs, hyperreflexia, and paresis of lower limbs and bilateral positive Babinski signs. Blood laboratory tests showed highly elevated acute phase proteins. Computed tomography (CT) of the neck showed atlanto-axial diffused and crown-like calcifications around the dens, which appeared largely eroded, but were kept in place by calcifications. No signs of atlanto-occipital and atlanto-axial subluxation were present (Fig. 1a, b). Numerous nodular-shaped calcium depositions were also present along the ligamenta flava, around the glenohumeral joints, the sternum, the pelvis, and within the small articulations of her hands (not shown). A diagnosis of severe systemic chondrocalcinosis with

D. Cammelli and G. Vitiello equally contributed to this work.

Gianfranco Vitiello

gianfranco.vitiello@unifi.it

1 Rheumatology Section, Immunoallergology Unit, Azienda Ospedaliero-Universitaria Careggi, Florence, Italy

2 Experimental and Clinical Medicine Department, University of Florence, Azienda Ospedaliero Universitaria Careggi, Largo Brambilla 3, 50134 Florence, Italy

3 Experimental and Clinical Biomedical Sciences Department, University of Florence, Florence, Italy

4 Medical-Geriatric Department, Azienda OspedalieroUniversitaria Careggi, Florence, Italy crowned dens syndrome (CDS) was made. Because of the clinical suggestion of medullar compression, she underwent magnetic resonance of the cervical tract, which showed anterior compression of the spinal cord by the pannus determined by the calcium deposition associated with hypersignal intensity on T2-weighted images at C1-C2 level (Fig. 1c). Bolus corticosteroids (methylprednisolone $500 \mathrm{mg} /$ daily) and oral colchicine (1 mg/daily) were administered for 1 week without any clinical improvement. Off-label subcutaneous anakinra (100 mg/daily) was added to therapy at day 6 , but the clinical situation did not ameliorate during the subsequent days. The patient died 16 days after the admission due to cardiorespiratory failure.

Crowned dens syndrome is a rare clinical manifestation of calcium pyrophosphate deposition disease. It is caused by the deposition of calcium pyrophosphate around the $\mathrm{C} 2$ vertebra, and usually manifests as acute severe neck pain, fever, and high levels of acute phase proteins [1]. For this reason, it is usually confused with meningitis, giant cell arteritis, polymyalgia rheumatica, or spondylitis [2]. According to a case series, neither the presence of jaw claudication, acute onset headache, nor a temporal artery biopsy is able to distinguish among these diseases. Only chondrocalcinosis around the $\mathrm{C} 2$ vertebra detected by $\mathrm{CT}$ scan seems to be pathognomonic for the diagnosis [2]. However, CT scan that detected chondrocalcinosis alone should not be used as sole diagnostic criteria in the absence of clinically evident arthritis [1]. Myelopathy is an extremely rare complication of CDS [3]. Therapy of CDS is based primarily on nonsteroidal anti-inflammatory drugs (NSAIDs); corticosteroids and colchicine seem to be effective in severe or recurrent cases [1,4]. Some case series support the effectiveness of interleukin 1-beta inhibitors in patients with acute arthritis due to calcium pyrophosphate deposition [5]. 

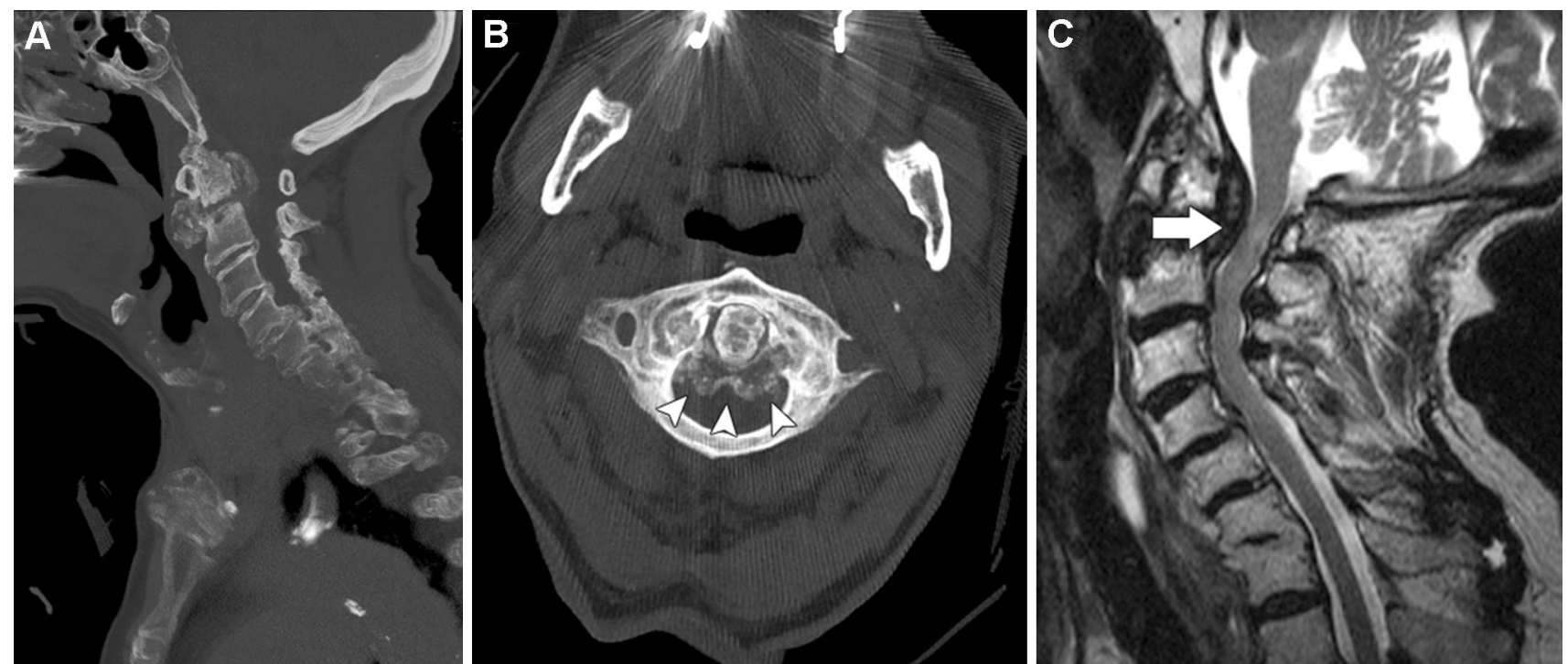

Fig. 1 Computed tomography (a, b) and T2-weighted magnetic resonance (c) of the cervical tract in a patient with severe CDS. a, b Atlanto-axial diffused and crown-like calcifications around the dens, which appeared largely eroded but still kept in place by

Febrile neck pain is a symptom that commonly prompts the patient to come to the emergency department. This case report aims to remind physician that CDS, although rare, has to be considered in the differential diagnosis of acute febrile neck pain, especially in the elderly. A CT scan of the cervical columna is a useful tool for the detection of chondrocalcinosis, and permits the avoidance of useless invasive procedures. Although anakinra (an interleukin 1-beta-inhibitor) has demonstrated effectiveness in the treatment of CDS, it seems to be ineffective in the resolution of the massive inflammation when myelopathy has already developed.

\section{Compliance with ethical standards}

Conflict of interest The authors declare that they have no conflict of interest.

Statement of human and animal rights All procedures performed in our study involving human participants are in accordance with the ethical standards of the institutional and/or national research calcifications. No sign of atlanto-occipital and atlanto-axial subluxation is present. c Anterior compression of the spinal cord by the pannus of the calcium deposition associated with hypersignal intensity at $\mathrm{C} 1-\mathrm{C} 2$ level

committee and with the 1964 Helsinki declaration and its later amendments or comparable ethical standards.

\section{Informed consent None.}

\section{References}

1. Rosenthal A, Lawrence M (2016) Calcium pyrophosphate deposition disease. N Engl J Med 374(26):2575-2584

2. Aouba A, Vuillemin-Bodaghi V, Mutschler C, De Bandt M (2004) Crowned dens syndrome misdiagnosed as polymyalgia rheumatica, giant cell arteritis, meningitis or spondylitis: an analysis of eight cases. Rheumatology 43(12):1508-1512

3. Ciricillo S, Weinstein P (1989) Foramen magnum syndrome from pseudogout of the atlanto-occipital ligament. J Neurosurg 71:141-143

4. Oka A, Okazaki K, Takeno A, Kumanomido S, Kusunoki R, Sato $S$ et al (2015) Crowned dens syndrome: report of three cases and a review of the literature. J Emerg Med 49(1):9-13

5. Ottaviani S, Brunier L, Sibilia J, Ardizzone M, Wendling D, Gill G induced arthritis: a report of 16 cases and review of the literature. Jt Bone Spine 80:178-182 et al (2013) Efficacy of anakinra in calcium pyrophosphate crystal- 